07

\title{
Влияние флуктуаций состава на излучательную рекомбинацию в узкозонных полупроводниковых твердых растворах
}

\author{
(C) А.В. Шиляев, ${ }^{1}$ К.Д. Мынбаев, ${ }^{1,2}$ Н.Л. Баженов, ${ }^{1}$ А.А. Грешнов ${ }^{1}$ \\ ${ }^{1}$ Физико-технический институт им. А.Ф. Иофрфе РАН, \\ 194021 Санкт-Петербург, Россия \\ ${ }^{2}$ Университет ИТМО, \\ 197101 Санкт-Петербург, Россия \\ e-mail: vozzdooh@gmail.com
}

(Поступило в Редакцию 5 апреля 2016 г.)

\begin{abstract}
Экспериментально исследована фотолюминесценция эпитаксиальных структур на основе узкозонных твердых растворов CdHgTe и установлено наличие в структурах масштабных флуктуаций состава, локализующих носители заряда. Предложена модель, описывающая влияние флуктуаций на скорость излучательной рекомбинации, форму спектров люминесценции и положение их пика. Модель описывает транспорт и рекомбинацию носителей в условиях сильной неоднородности состава твердого раствора и показывает, как локализация носителей проявляется в особенностях спектров люминесценции.
\end{abstract}

DOI: $10.21883 /$ JTF.2017.03.44249.1832

\section{Введение}

Стохастический характер распределения атомов по узлам кристаллической решетки полупроводниковых твердых растворов приводит к появлению хвостов плотности состояний, что существенно уменьшает ширину запрещенной зоны $E_{g}$, проявляющуюся в оптических экспериментах. Низкая плотность таких состояний делает их трудноразличимыми при исследовании оптического поглощения, однако в фото- и электролюминесценции хвосты плотности состояний играют важную роль из-за процессов неупругого рассеяния, приводящих к энергетической релаксации и накоплению носителей в минимумах потенциальной энергии. При низких температурах пики люминесценции оказываются смещены в красную область относительно края поглощения или номинального (расчетного) значения ширины запрещенной зоны полупроводника. С повышением температуры этот сдвиг уменьшается, так как из-за термического возбуждения носителей, рекомбинация которых определяет эффект излучения, они перестают быть локализованными во флуктуациях.

Влияние флуктуаций состава на оптические свойства изучалось для многих полупроводниковых материалов и гетероструктур (см., например, [1-5]). Особый интерес оно представляет для узкозонных полупроводников, поскольку при типичной для них величине $E_{g}$ в 100-300 meV энергетические флуктуации амплитудой в десятки $\mathrm{meV}$, обычные для твердых растворов, составляют не единицы, а десятки процентов от $E_{g}$ и неизбежно должны оказывать существенное влияние на свойства материалов. Поскольку подобные материалы широко применяются в инфракрасной фото- и оптоэлектронике, исследование флуктуаций химического состава в них представляет не только научный, но и практический интерес. В настоящей работе мы анализируем результаты исследований люминесценции узкозонных полупроводниковых твердых растворов и представляем модель для описания влияния флуктуаций состава на люминесценцию.

\section{Экспериментальные результаты}

Для экспериментальных исследований были выбраны гетероэпитаксиальные структуры на основе твердых растворов $\mathrm{Cd}_{x} \mathrm{Hg}_{1-x} \mathrm{Te}$ (КРТ). Эти твердые растворы являются основным материалом для изготовления фотоприемников с предельной чувствительностью среднего $(2-5 \mu \mathrm{m})$ и дальнего $(8-14 \mu \mathrm{m})$ инфракрасных диапазонов [6]. Величина $E_{g}$ КРТ может изменяться при температуре $T=300 \mathrm{~K}$ от 0 до $1.6 \mathrm{eV}$.

Исследовалась фотолюминесценция (ФЛ) структур в диапазоне $4.2<T<300 \mathrm{~K}$ при возбуждении полупроводниковым лазером $\mathrm{InGaAs} / \mathrm{GaAs}$ с длиной волны $1.03 \mu \mathrm{m}$ с частотой следования импульсов $1 \mathrm{kHz}$ и их длительностью $1 \mu \mathrm{s}$. Спектры ФЛ записывались на автоматизированной установке с монохроматором МДР-23. Сигнал ФЛ регистрировался фотодиодом на основе $\mathrm{Ge}$ или охлаждаемым фотодиодом из InSb. Возбуждение сигнала осуществлялось со стороны эпитаксиальной структуры, а регистрация - со стороны подложки, на которой она была выращена. Структуры были выращены методами молекулярно-лучевой эпитаксии (МЛЭ) и жидкофазной эпитаксии (ЖФЭ). Толщина слоя КРТ, из которого собирался сигнал ФЛ, составляла от 5 до $9 \mu \mathrm{m}$. Химический состав $x$ этого слоя в структурах, выращенных МЛЭ, измерялся с помощью эллипсометрии in situ [7]. Дополнительный контроль $x$ в этих образцах, а также определение $x$ в структурах, выращенных ЖФЭ, проводились ex situ на основе измерений спектров 
оптического пропускания. Образцы для исследования ФЛ имели площадь $\sim 1 \mathrm{~cm}^{2}$.

Оригинальные экспериментальные результаты исследования ФЛ различных структур на основе КРТ были представлены нами в работах [8-12]. Пример результатов представлен на рис. 1, $a$, где приведены нормализованные спектры ФЛ трех структур, записанные при $T=4.2 \mathrm{~K}$, а также их аппроксимация (тонкие черные линии). Спектр 1 соответствует структуре с номинальным (по данным эллипсометрии и оптического пропускания) составом $x=0.36$, выращенной МЛЭ на подложке из $\mathrm{Si}(\mathrm{CdHgTe} / \mathrm{Si})$. Спектр хорошо описывался гауссовым распределением, полуширина (полная ширина на половине высоты) спектра составляла $5.7 \mathrm{meV}$. Спектр 2 соответствует структуре с номинальным составом $x=0.38$, выращенной МЛЭ на подложке из $\mathrm{GaAs}(\mathrm{CdHgTe} / \mathrm{GaAs})$. Этот спектр также хорошо описывался гауссовым распределением, его полуширина составляла $16.3 \mathrm{meV}$. Спектр 3 также соответствует структуре с номинальным составом $x=0.38$, но данная структура была выращена методом ЖФЭ на подложке $\mathrm{CdTe}(\mathrm{CdHgTe} / \mathrm{CdTe})$. Обращает на себя внимание сдвиг спектра 3 в коротковолновую область относительно спектра 2 при том, что образцы имели один и тот же химический состав. Спектр 3 содержал две линии, и полуширина высокоэнергетической линии, которую, как и одиночные линии спектров 1 и 2 , можно было сопоставить с „межзонным“ излучением, составляла $5.0 \mathrm{meV}$. Присутствие второй (низкоэнергетической) линии было обусловлено высокой концентрацией в рассматриваемом материале вакансий ртути - акцепторов в КРТ [9].

На рис. $1, b$ представлены температурные зависимости энергетического положения пика линии межзонного излучения $E_{\mathrm{PL}}$ для трех структур КРТ с составом $x=0.38$. Здесь же представлена расчетная зависимость величины $E_{g}(T)$ для данного состава, полученная с использованием эмпирического выражения из работы [13],

$$
\begin{aligned}
E_{g}= & -0.303(1-x)+1.606 x-0.132 x(1-x) \\
& +\frac{4.95(1-x)+3.25 x-3.93 x(1-x)}{11(1-x)+78.7 x+T} T^{2} 10^{-4} .
\end{aligned}
$$

Как видно из рис. $1, b$, при низкой температуре наблюдается значительное расхождение $E_{\mathrm{PL}}$ и расчетного значения $E_{g}$, а с повышением температуры это расхождение исчезает. Для структур на основе КРТ это обычно объясняется тем, что „межзонная“ ФЛ представляет собой излучение, обусловленное рекомбинацией экситонов, локализованных на флуктуациях химического состава твердого раствора [8-12,14-17]. При этом разница между $E_{\mathrm{PL}}$ и $E_{g}$ прямо или опосредованно должна определять некую условную энергию локализации экситона и соответственно характеризовать масштаб флуктуаций [15-17]. Последнюю величину также должна характеризовать и полуширина линии излучения. Если
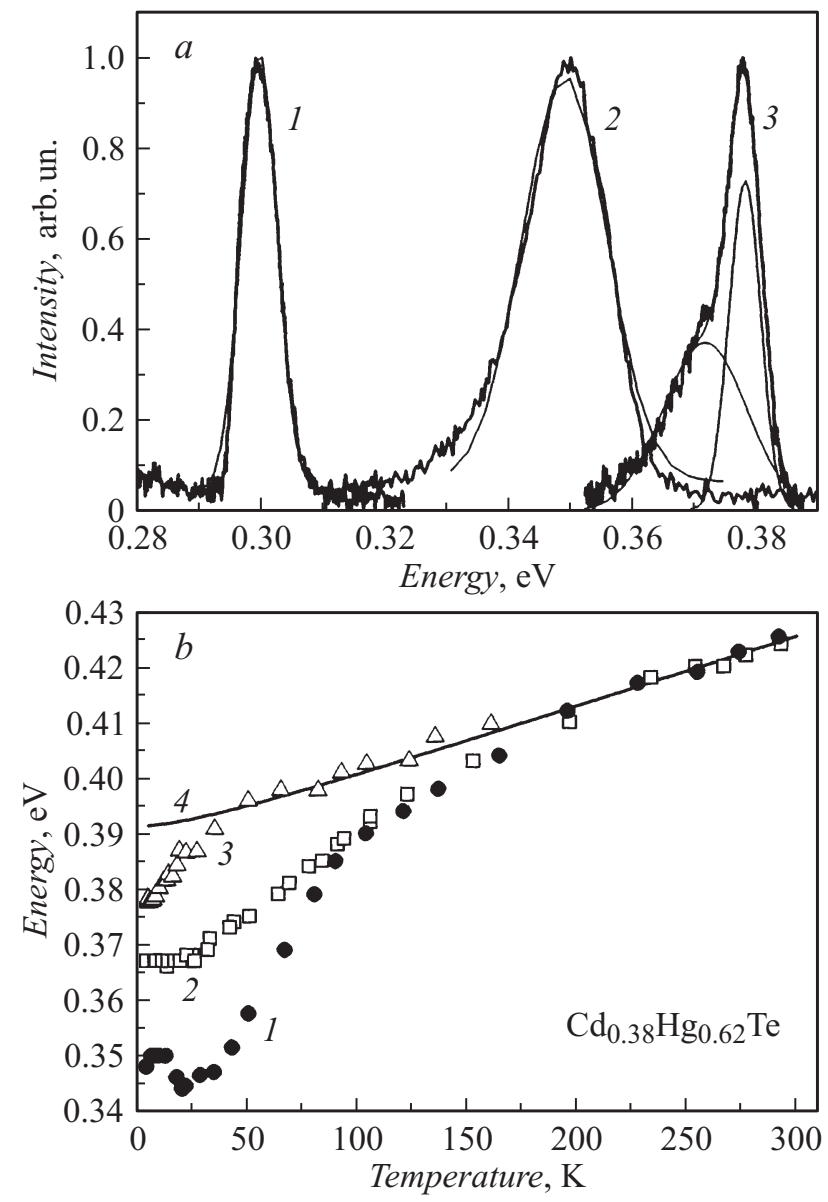

Рис. 1. Спектры ФЛ гетероэпитаксиальных структур на основе КРТ при $T=4.2 \mathrm{~K}, a-$ структуры $\mathrm{CdHgTe} / \mathrm{Si}$ с $x=0.36(1)$ и двух структур с $x=0.38$, выращенных - МЛЭ $\mathrm{CdHgTe} / \mathrm{GaAs}(2)$ и ЖФЭ $\mathrm{CdHgTe} / \mathrm{CdTe}(3)$, тонкими линиями показана аппроксимация спектров и отдельных линий гауссовым распределением; $b$ - экспериментальные температурные зависимости положения пиков ФЛ для трех структур на основе КРТ с $x=0.38$ : МЛЭ $\mathrm{CdHgTe} / \mathrm{GaAs}(1)$, МЛЭ $\mathrm{CdHgTe} / \mathrm{Si}(2)$ и ЖФЭ $\mathrm{CdHgTe} / \mathrm{CdTe}(3)$, и расчетная зависимость $E_{g}(x, T)$ для КРТ данного состава (4).

руководствоваться этими представлениями, то на основании данных рис. $1, b$ можно сделать вывод, что образец, выращенный ЖФЭ, характеризуется наименьшим размахом флуктуаций, а образец, выращенный МЛЭ на подложке GaAs, - наибольшим, поскольку все образцы имеют один и тот же состав $\mathrm{x}=0.38$. При этом, как следует из данных рис. 1 , полуширины линий ФЛ (5-17 meV) гораздо меньше разницы между $E_{g}$ и $E_{\mathrm{PL}}(14-45 \mathrm{meV})$, хотя согласно „классической“ модели [18], описывающей разупорядочение полупроводниковых твердых растворов, для одной и той же структуры они должны быть близки.

На рис. 2 обобщены полученные из наших экспериментов данные по величине $E_{g}-E_{\mathrm{PL}}$ (символы 1,3 и 5) и полуширине спектральной линии ФЛ, соответствовавшей межзонному излучению (символы 2,4 


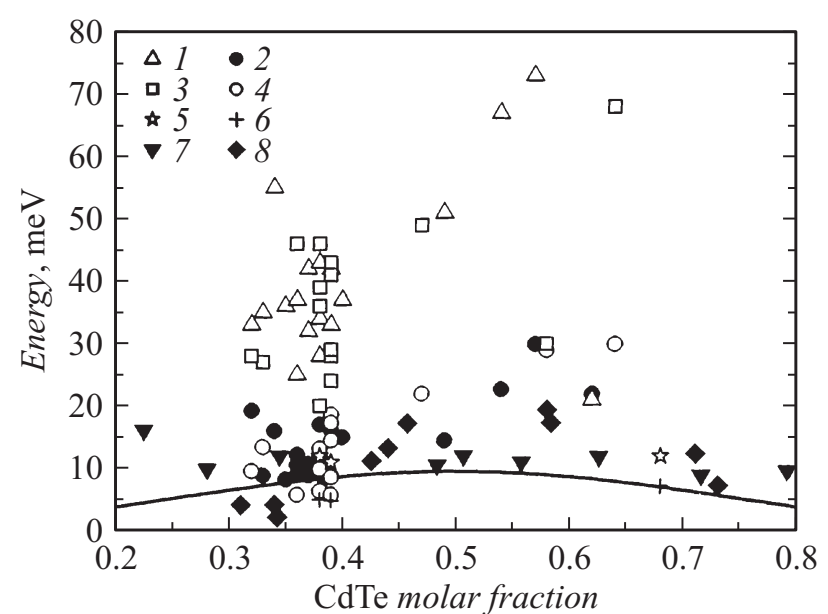

Pис. 2. Экспериментальные (символы) и расчетная (согласно модели [18], сплошная линия) зависимости условной „энергии локализации экситона“ (носителей заряда), символы $1,3,5,7$ и 8 , и полуширины линии межзонного излучения (символы 2 , 4 и 6) по данным ФЛ от состава твердого раствора: $1,3-$ МЛЭ $\mathrm{CdHgTe} / \mathrm{GaAs}, 2,4$ - МЛЭ $\mathrm{CdHgTe} / \mathrm{Si}, 5,6$ - ЖФЭ $\mathrm{CdHgTe/CdTe,} 7-$ МДН и ЖФЭ по данным [16] и $8-$ МДН по данным [17].

и 6), в зависимости от состава твердого раствора. Также на этом рисунке приведена зависимость „размаха“ флуктуаций в твердом растворе КРТ от его состава, полученная в рамках модели [18] (сплошная линия), и значения энергии, характеризующие разупорядочение в КРТ по данным экспериментов по ФЛ, имеющимся в литературе. В частности, символами 7 показаны данные по энергии локализации экситона (носителей заряда) в образцах КРТ, выращенных методом движущегося нагревателя (МДН, рост объемных кристаллов) и ЖФЭ из работы [16] (энергия локализации оценивалась по модели [19]), а символами 8 - данные по величине $E_{g}-E_{\mathrm{PL}}$ при $T=30 \mathrm{~K}$ из работы [17], полученные на кристаллах, выращенных МДН. В силу использования различных моделей для оценки величины энергии локализации экситона, различных зависимостей $E_{g}(x, T)$ и различной температуры записи спектров точное количественное сопоставление литературных данных с результатами наших экспериментов не представляется возможным. Однако можно сделать некоторые качественные выводы, и главным из них, очевидно, будет подтверждение того факта, что образцы КРТ, выращенные МДН и ЖФЭ, характеризуются гораздо менее масштабными флуктуациями состава, чем образцы, выращенные МЛЭ. Также отметим, что в соответствии с имеющимися представлениями, если говорить о стохастическом характере распределения атомов между различными узлами решетки кристаллов полупроводниковых твердых растворов, максимальный масштаб флуктуаций и соответственно наибольшая энергия локализации экситона должны наблюдаться для твердых растворов с $x=0.50$. Результаты, полученные на образцах, выращенных МДН и ЖФЭ, в целом следуют этой тенденции, в то время как об образцах, выращенных МЛЭ, этого сказать нельзя, здесь размах флуктуаций возрастает с увеличением состава вплоть до $x \sim 0.63$. Наконец, обращает на себя внимание большой разброс данных по величинам $E_{g}-E_{\mathrm{PL}}$ и полуширинам линий излучения для образцов КРТ, выращенных МЛЭ, близкого состава. Все эти результаты говорят о том, что разупорядочение, имеющее место в образцах КРТ, выращенных МЛЭ, является не стохастическим, а вызванным особенностями технологии, возможно, неравновесным характером МЛЭ [15-17]. Отметим, что для ряда полупроводниковых твердых растворов, в частности InGaN, такое „технологическое“ разупорядочение изучено уже весьма подробно (см., например, [20]). Более того, в структурах на основе выращенных МЛЭ твердых растворов $\mathrm{AlGaN}$, для которых изначально характерна гораздо более высокая степень упорядочения, чем для InGaN, неоднородности состава, локализующие экситоны, создаются при помощи особых технологических приемов искусственно - для увеличения квантового выхода излучения $[21,22]$.

Обобщая представленные на рис. 1 и 2 данные, констатируем, что для исследованных структур КРТ, выращенных МЛЭ, экспериментально выявлено значительное разупорядочение, масштаб которого для одного и того же состава твердого раствора - по-видимому, в зависимости от особенностей технологических режимов выращивания структур - мог отличаться в несколько раз. Это позволило допустить значительную свободу при моделировании, в частности произвольное задание масштаба флуктуаций.

\section{Моделирование и обсуждение результатов}

Несмотря на наличие большого объема экспериментальных данных и проработанность общих теоретических представлений о влиянии неоднородностей состава на оптические и электрические свойства полупроводниковых твердых растворов, на практике добиться количественного соответствия экспериментальных данных и теории не так просто. Адекватное теоретическое описание явлений в этой области требует учета целого ряда физических процессов, происходящих с носителями заряда с момента их инжекции в систему и до рекомбинации, излучательной или безызлучательной. Конечный результат (интенсивность люминесценции и вид спектров) зависит от подробностей кинетики носителей, определяемой тем, как именно электроны и дырки „впрыскиваются“ в систему, их рассеяния на индуцируемом флуктуациями состава случайном потенциале, неупругого рассеяния на оптических и акустических фононах, приводящих к энергетической релаксации в минимумы потенциала, характеристик процессов излучательной рекомбинации и безызлучательной рекомбинации с предварительным захватом на глубокие центры. Учет всех этих 

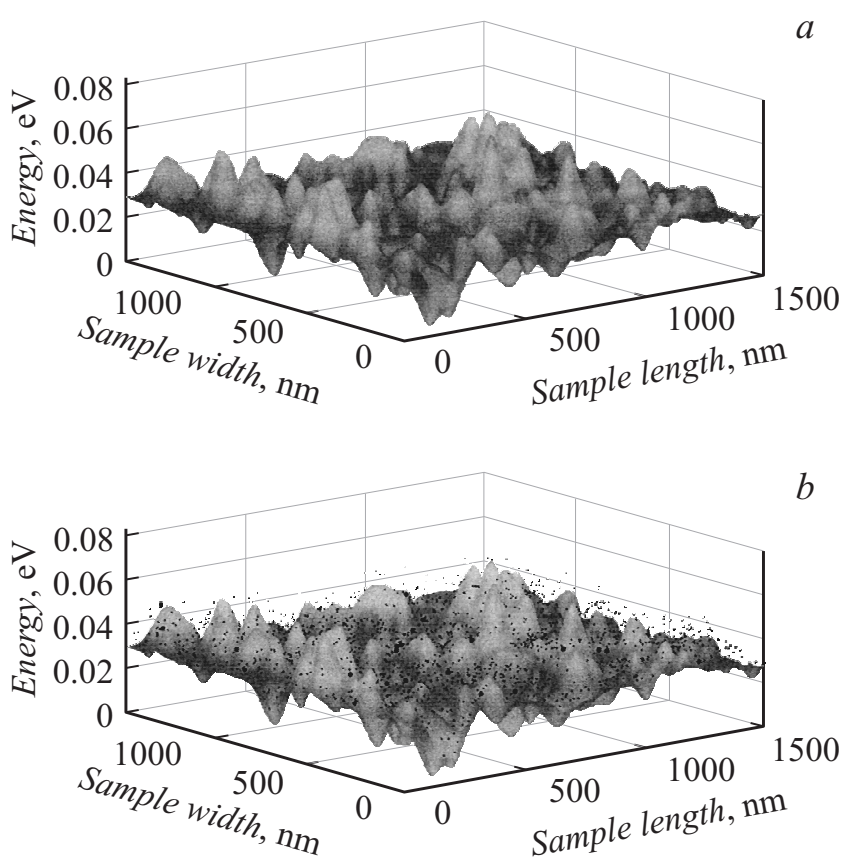

Pис. 3. Трехмерное изображение потенциального рельефа без носителей $(a)$ и потенциальный рельеф с инжектированными носителями (точки) и дефектами (жирные точки) после релаксации $(b)$.

процессов в существенно неоднородной системе требует проведения численных расчетов (моделирования) для конкретных реализаций случайного распределения флуктуаций состава. Строго говоря, моделирование должно быть квантово-механическим, поскольку иначе описать процессы взаимодействия электронов с фотонами и фононами не представляется возможным. В реальности проведение квантово-механических расчетов для скольлибо протяженной системы, содержащей хотя бы десятки минимумов случайного потенциала, выходит за пределы любых вычислительных возможностей. Кроме того, рассматриваемая задача в целом является по существу квазиклассической, поскольку о когерентных состояниях при рассматриваемых температурах речи не идет. Поэтому нами разработана гибридная модель, в которой состояние каждого носителя описывается гауссовым волновым пакетом, содержащим координаты и импульсы частицы в качестве параметров (заметим, что и в импульсном представлении волновая функция такого пакета имеет гауссов вид):

$$
\Psi_{\mathbf{r}_{0} \mathbf{k}_{0}}(\mathbf{r})=\frac{1}{\sqrt{\pi a}} e^{i \mathbf{k}_{0}\left(\mathbf{r}-\mathbf{r}_{0}\right)-\frac{\left(\mathbf{r}-\mathbf{r}_{0}\right)^{2}}{2 a^{2}}}
$$

Разработанная нами модель реализована в программe MatLAB для двумерного случая, как мы считаем, качественно не отличающегося от трехмерного ввиду квазиклассического режима, и ниже мы кратко опишем этапы моделирования. Прежде всего генерируется потенциал $U(\mathbf{r})$, который представляет собой суперпозицию трехмерных гауссианов, случайно распределенных по поверхности образца, рис. $3, a$ (заметим, что в случае флуктуаций состава твердого раствора вид случайного потенциала для электронов и дырок идентичный, отличается только амплитуда). Инжекция носителей описывается в виде равноотстоящих по времени событий добавления одного электрона и одной дырки в случайные точки системы со скоростями, обеспечивающими кинетическую энергию на уровне порядка энергии оптического фонона, что позволяет не рассматривать период быстрой энергетической релаксации на оптических фононах. Далее с определенным шагом по времени (несколько меньшим времени упругого рассеяния) просчитывается классическая динамика волновых пакетов, представляющих состояния электронов и дырок (т.е. эволюциях их параметров $\mathbf{r}$ и $\mathbf{k}=\mathbf{p} / \hbar$ в соответствии со вторым законом Ньютона) в рассматриваемой реализации случайного потенциала, и энергетическая релаксация на акустических фононах. При этом для расчета числа состояний на единицу фазового объема системы мы поделили фазовое пространство на гиперкубы со сторонами $\left(\sqrt{2 \pi} a, \sqrt{2 \pi} a, \sqrt{2 \pi} a^{-1}, \sqrt{2 \pi} a^{-1}\right)$ с объемом $(2 \pi)^{d}(d=2)$ каждый и договорились относить частицу в состоянии (2) к элементу фазового пространства с номером $n=\left(n_{x}, n_{y}, n_{k_{x}}, n_{k_{y}}\right)$, если

$$
\begin{gathered}
n_{x}=\left[\frac{x}{\sqrt{2 \pi} a}\right], n_{y}=\left[\frac{y}{\sqrt{2 \pi} a}\right], n_{k_{x}}=\left[\frac{k_{x}}{\sqrt{2 \pi} a^{-1}}\right], \\
n_{k_{y}}=\left[\frac{k_{y}}{\sqrt{2 \pi} a^{-1}}\right],
\end{gathered}
$$

где выражение в квадратных скобках - целая часть числа. Выполнение принципа Паули, т.е. недопустимость сосуществования внутри одного элемента фазового объема двух носителей одного типа с одинаковым спином, обеспечивалось: 1) учетом степени заполнения состояний при расчете вероятности неупругих процессов с участием фононов, 2) введением фиктивного расталкивания между электронами, близко находящимися в фазовом пространстве (т.е. в пределах одного гиперкуба; на самом деле между ними присутствует и весьма реальное - кулоновское - отталкивание). Выбор соотношения между сторонами элементарного гиперкуба в реальном и фазовом пространстве, т. е. параметра $a$ в формуле (2), диктовался компромиссом между точностью в определении положения частицы (меньше масштабов изменения случайного потенциала $U(r))$ и точностью определения кинетической энергии (существенно меньше температуры и квазиуровня Ферми), типичные использованные значения для электронов и дырок составляли $a_{e}=30 \mathrm{~nm}, a_{h}=10 \mathrm{~nm}$.

Базовая адекватность предлагаемой модели контролировалась расчетами контрольных характеристик в случае $U(\mathbf{r})=0$. В частности, известное соотношение между концентрацией $n$ и уровнем химического потенциала $\mu$ в пределе нулевой температуры

$$
n=2 \int \frac{d^{2} k}{(2 \pi)^{2}} f(E(\mathbf{k}), \mu, T=0)=\frac{k_{F}^{2}}{2 \pi}=\frac{m^{*} \mu}{\pi \hbar^{2}}
$$


может быть легко получено и на языке волновых пакетов

$$
\begin{aligned}
n & =\frac{2}{S} \Sigma_{n} f\left(E_{n}, \mu, T=0\right) \\
& =\frac{2}{S} \frac{S}{2 \pi a^{2}} \Sigma_{n_{k_{x}}, n_{k_{y}}} f\left(\frac{\pi \hbar^{2}\left(n_{k_{x}}^{2}+n_{k_{y}}^{2}\right)}{m^{*} a^{2}}, \mu, T=0\right) \\
& \approx \frac{1}{\pi a^{2}} \int d n_{k_{x}} d n_{k_{y}} \theta\left(\mu-\frac{\pi \hbar^{2}\left(n_{k_{x}}^{2}+n_{k_{y}}^{2}\right)}{m^{*} a^{2}}\right)=\frac{m^{*} \mu}{\pi \hbar^{2}} .
\end{aligned}
$$

Из выражения для интеграла перекрытия двух волновых пакетов вида (2)

$$
\begin{aligned}
I_{12} & =\left|\int d^{2} r \Psi_{r_{1}, k_{1}}^{*}(\mathbf{r}) \Psi_{r_{2}, k_{2}}(\mathbf{r})\right|^{2} \\
& =\left|e^{-\frac{\left(\mathbf{r}_{1}-\mathbf{r}_{2}\right)^{2}}{4 a^{2}}-\frac{\left(\mathbf{k}_{1}-\mathbf{k}_{2}\right)^{2} a^{2}}{4}+\frac{i}{2}\left(\mathbf{r}_{1}-\mathbf{r}_{2}\right)\left(\mathbf{k}_{1}+\mathbf{k}_{2}\right)}\right|^{2} \\
& =e^{-\frac{\left(\mathbf{r}_{1}-\mathbf{r}_{2}\right)^{2}}{2 a^{2}}-\frac{\left(\mathbf{k}_{1}-\mathbf{k}_{2}\right)^{2} a^{2}}{2}}
\end{aligned}
$$

следует, что для двух пакетов, находящихся в центрах соседних гиперкубов (т.е. разделенных расстоянием $\sqrt{2 \pi} a$ или волновым вектором $\left.\sqrt{2 \pi} a^{-1}\right)$, величина квадрата интеграла перекрытия достаточно мала, $I_{12}=e^{-\pi} \approx 0.05$. Иначе говоря, существенное перекрытие пакетов (и соответственно сильное „контактное“ взаимодействие) достигается только в пределах одного элементарного объема фазового пространства, а далее оно экспоненциально спадает, что еще раз обосновывает применяемый подход.

Далее мы учитываем возможность рекомбинации электронов и дырок в результате излучательных и безызлучательных процессов. Для вычисления вероятностей переходов из заполненного состояния в зоне проводимости в пустое состояние в валентной зоне с испусканием фотона воспользуемся золотым правилом Ферми

$$
\begin{aligned}
W_{1 \rightarrow 2}= & \frac{2 \pi}{\hbar} \Sigma_{\mathbf{q}, v}\left|\left\langle 1_{\mathbf{q}, v} ; v, \mathbf{r}_{2}, \mathbf{k}_{2}\left|\mathscr{H}^{\prime}\right| 0_{\mathbf{q}, v} ; c, \mathbf{r}_{1}, \mathbf{k}_{1}\right\rangle\right|^{2} \\
& \times \delta\left(E_{g}+\varepsilon_{e}\left(k_{1}\right)+\varepsilon_{h}\left(\mathbf{k}_{2}\right)-\hbar \omega_{\mathbf{q}}\right),
\end{aligned}
$$

где внутризонные энергии электрона $\varepsilon_{e}$ и дырки $\varepsilon_{h}$ отсчитываются в направлении „от запрещенной зоны“, $\omega_{\mathbf{q}}=c q$, а оператор взаимодействия $\mathscr{H}$ после учета чисел заполнения фотонов и отделения блоховских функций, т.е. в представлении плавных огибающих (2), имеет вид

$$
\mathscr{H}^{\prime}=\frac{e \sqrt{2 \pi} \hbar \mathbf{e}_{v} \mathbf{p}_{c v}}{m_{0} n_{\omega} \sqrt{\omega_{q}} \sqrt{V}} e^{-i \mathbf{q r}}
$$

где $\mathbf{e}_{v}$ - один из двух возможных ортов поляризации, $n_{\omega}$ - показатель преломления, $\mathbf{p}_{c v}-$ матричный элемент оператора импульса между блоховскими функциями $u_{c}$ и $u_{v}, V-$ нормировочный объем. Поскольку импульсом фотона q можно пренебречь, квадрат матричного элемента $e^{-i \mathbf{q r}}$ сводится к (6). После суммирования по q

$$
\Sigma_{\mathbf{q}} \delta\left(\frac{\hbar c q}{n_{\omega}}-\varepsilon\right)=\int \frac{V d^{3} q}{(2 \pi)^{3}} \delta\left(\frac{\hbar c q}{n_{\omega}}-\varepsilon\right)=\frac{V n_{\omega}^{3} \varepsilon^{2}}{2 \pi^{2} \hbar^{3} c^{3}}
$$

получаем

$$
W_{1 \rightarrow 2}=\frac{4 e^{2} n_{\omega} \varepsilon_{12}\left|\mathscr{P}_{c v}\right|^{2}}{\hbar^{2} m_{0}^{2} c^{3}} e^{-\frac{\left(\mathbf{r}_{1}-\mathbf{r}_{2}\right)^{2}}{2 a^{2}}-\frac{\left(\mathbf{k}_{1}-\mathbf{k}_{2}\right)^{2} a^{2}}{2}} .
$$

Здесь $\varepsilon_{12}=E_{g}+\varepsilon_{e}\left(\mathbf{k}_{1}\right)+\varepsilon_{h}\left(\mathbf{k}_{2}\right)$, a $\left|\mathscr{P}_{c v}\right|=\left|e_{\nu} \mathbf{p}_{c v}\right|$ мы ввели, считая результат одинаковым для любой поляризации $v$ (изотропия). В случае отличающихся ширин волновых пакетов для электронов и дырок, для перекрытия волновых пакетов (6) и соответственно для экспоненциального множителя в правой части (10) имеем

$$
I_{12}=\left(\frac{2 a_{e} a_{h}}{a_{e}^{2}+a_{h}^{2}}\right)^{2} e^{-\frac{\left(\mathbf{r}_{1}-\mathbf{r}_{2}\right)^{2}}{a_{e}^{2}+a_{h}^{2}}-\frac{a_{e}^{2} a_{h}^{2}\left(\mathbf{k}_{1}-\mathbf{k}_{2}\right)^{2}}{a_{e}^{2}+a_{h}^{2}}} .
$$

При описании безызлучательной рекомбинации мы исходили из предположения, что ее главным каналом является рекомбинация на глубоких центрах, переход на которые осуществляется благодаря существованию промежуточных состояний вида „электрон/дырка в запрещенной зоне + локальное колебание атомов решетки“ [23]. Оговоримся, что, хотя данное приближение является вполне допустимым для упомянутых выше нитридных материалов, для твердых растворов КРТ оно применимо с определенными оговорками. Традиционно считается, что в этих материалах при высоких температурах в качестве безызлучательной доминирует ожерекомбинация [24]. Тем не менее для низких температур наша модель оказывается вполне применимой, например, как показали уточненные расчеты, для КРТ c $x \geq 0.3$ излучательная рекомбинация доминирует от низких температур вплоть до $T=230 \mathrm{~K}$ [25]. Поскольку детальное описание процесса захвата на глубокий центр представляет собой сложную задачу [23], в нашей модели используется упрощенное описание, отражающее, однако, особенности процесса. Для носителей с достаточно большой кинетической энергией зацепление за локальные колебания маловероятно, поэтому мы считаем, что ненулевая вероятность захвата возникает лишь при попадании достаточно низкоэнергетичного электрона или дырки непосредственно в область дефекта. Если событие захвата наступило, частица продолжает оставаться на дефекте вплоть до того момента, когда на этот же дефект тем же путем попадает носитель с противоположным зарядом.

В процессе энергетической релаксации носители стремятся занять самое выгодное энергетическое положение, располагаясь ближе к дну потенциального рельефа. При достаточно большой амплитуде флуктуаций, оказавшись внутри потенциальной ямы, носители уже не могут выбраться обратно (вероятность этого мала), т.е. локализуются в области локального минимума потенциальной энергии (рис. 3,b). Учитывая, что минимумы 
потенциальной энергии реализуются одновременно для электронов и дырок, вероятность их сближения повышается, а значит, растет вероятность излучательной рекомбинации. В то же время дефекты материала распределяются случайным образом вне зависимости от формы потенциального рельефа и могут оказаться как в области ямы (в такой яме эффективность центра безызлучательной рекомбинации будет выше), так и в области „холма“ (в этом случае центр будет бездействовать). Таким образом, при увеличении амплитуды флуктуаций средняя эффективность центров безызлучательной рекомбинации падает, а отношение скоростей излучательной и безызлучательной рекомбинации растет, что и объясняет увеличение эффективности люминесценции в системах с выраженными неоднородностями состава, наблюдавшееся экспериментально [20-22].

В связи с важностью факта локализации носителей на минимумах потенциала, остановимся подробнее на особенностях процесса энергетической релаксации. Формула для темпа спонтанных переходов из произвольного электронного состояния 1 в состояние 2 с излучением фонона в изотропном приближении имеет вид [23]

$$
P_{1 \rightarrow 2}=\frac{\pi E_{D}^{2}}{\rho s} \int \frac{d^{3} k}{(2 \pi)^{3}} k\left|I_{12}(\mathbf{k})\right|^{2} \delta\left(E_{1}-E_{2}-\hbar s k\right),
$$

где $s-$ скорость звука, $\rho$ - плотность кристалла, $E_{D}$ - деформационный потенциал, $I_{21}(\mathbf{k})$ - матричный элемент плоской волны (волновой функции фонона) между двумя электронными состояниями вида (1):

$$
\begin{aligned}
I_{12}(\mathbf{k}) & =\left|\int d^{2} r \Psi_{\mathbf{r}_{2}, \mathbf{k}_{2}}^{*}(\mathbf{r}) e^{-i \mathbf{k} \mathbf{r}} \Psi_{\mathbf{r}_{1}, \mathbf{k}_{1}}\right|^{2} \\
& =e^{-\left(\mathbf{r}_{1}-\mathbf{r}_{2}\right)^{2}} 2 a^{2}-\frac{\left(\mathbf{k}_{1}-\mathbf{k}_{2}-\mathbf{k}\right)^{2} a^{2}}{2} .
\end{aligned}
$$

Дельта-функция (закон сохранения энергии) позволяет произвести интегрирование по $k_{z}-$ компоненте волнового вектора фонона, перпендикулярной рассматриваемому двумерному слою:

$$
\begin{aligned}
& P_{1 \rightarrow 2}=\frac{\pi E_{D}^{2}}{\rho s} \int \frac{d^{2} q}{(2 \pi)^{2}}\left|I_{21}(\mathbf{q})\right|^{2} \\
& \times \int_{-\infty}^{\infty} \frac{d k_{z}}{2 \pi}\left(E_{1}-E_{2}-\hbar s \sqrt{q^{2}+k_{z}^{2}}\right) \\
& =\frac{E_{D}^{2} Q_{12}^{2}}{\rho \hbar s^{2}} e^{-\frac{\mathbf{r}_{1}-\mathbf{r}_{2}}{2 a^{2}}} \int \frac{d^{2} q}{(2 \pi)^{2}} \frac{e^{-\frac{1}{2}\left(\mathbf{k}_{12}-\mathbf{q}\right)^{2} a^{2}}}{\sqrt{Q_{12}^{2}-q^{2}}},
\end{aligned}
$$

где $k_{12}=k_{1}-k_{2}$, а $Q_{12}=\left|E_{1}-E_{2}\right| /(\hbar s)$. Поскольку в реальном эксперименте толщина излучающих слоев составляла несколько микрометров, т.е. соответствовала трехмерному случаю, при сопоставлении с двумерной моделью мы пренебрежем величиной $q^{2}$ под корнем в формуле (14), после чего интеграл берется до конца и

$$
P_{1 \rightarrow 2} \approx \frac{E_{D}^{2}\left|E_{1}-E_{2}\right|}{2 \pi \hbar^{2} s^{3} \rho a^{2}} e^{-\frac{\left(\mathbf{r}_{1}-\mathbf{r}_{2}\right)^{2}}{2 a^{2}}} .
$$
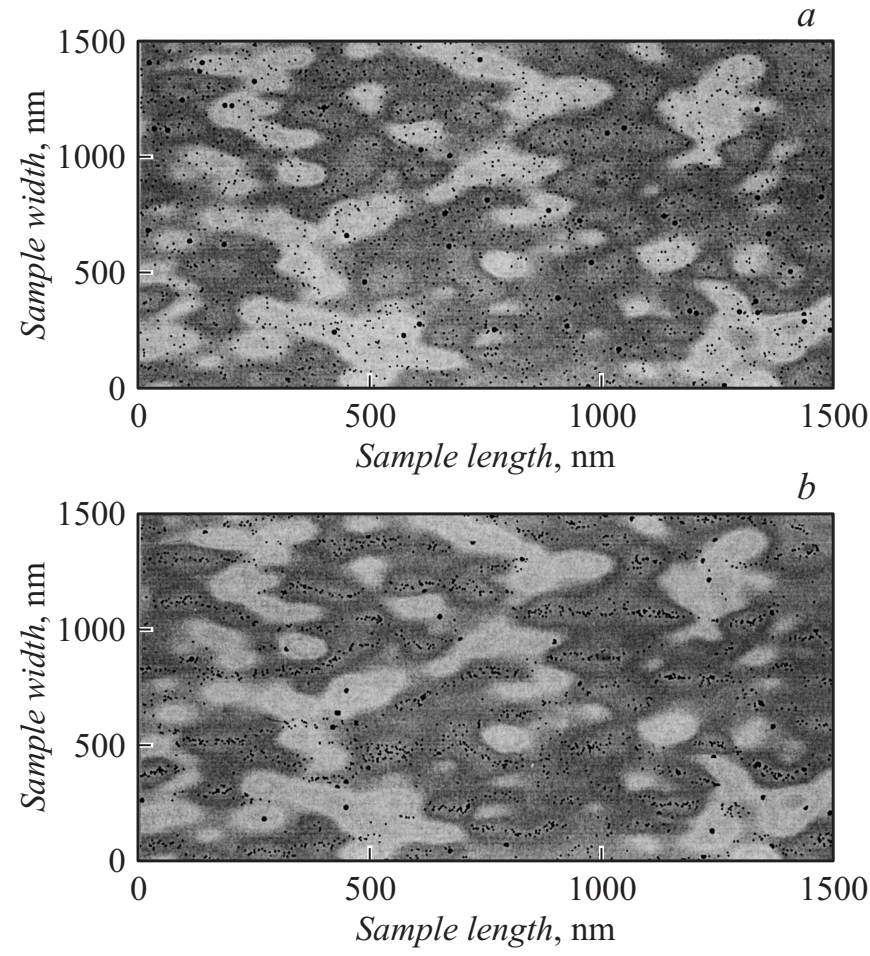

Рис. 4. Изображения потенциального рельефа с носителями после релаксации для различных амплитуд флуктуаций (вид „сверху“), амплитуды 0.155 (a) и $0.061 \mathrm{eV}(b)$.

В таком приближении вероятность переходов между электронными состояниями определяется лишь их координатами, а соотношение импульсов не играет роли, т. е. мы предположили, что излишек импульса всегда унесется фононом. Для учета статистики фононов введем функцию распределения Бозе-Эйнштейна для энергии, соответствующей переходу между некоторыми электронными состояниями 1 и 2 ,

$$
n_{12}=\frac{1}{\exp \left(\frac{\left|E_{1}-E_{2}\right|}{T}\right)-1} .
$$

Выражение для эффективного темпа переходов, учитывающее процессы поглощения, спонтанного и вынужденного излучений фононов, имеет вид:

$$
\tilde{P}_{1 \rightarrow 2}=\left[\left(1+n_{12}\right) \theta\left(E_{1}-E_{2}\right)+n_{12} \theta\left(E_{2}-E_{1}\right)\right] P_{1 \rightarrow 2},
$$

где $\theta(x)$ - ступенчатая функция Хевисайда.

На рис. 4 представлены два изображения потенциального рельефа с носителями после релаксации, вид „сверху“. Для случая, представленного на рис. 4, $a$, амплитуда флуктуаций была в $\sim 2.5$ раза больше, чем для случая рис. $4, b$. Сопоставляя результаты моделирования, констатируем, что для случая рис. 4, a существенная часть носителей сконцентрировалась в потенциальных ямах (более темные области), в то же время в областях локальных максимумов потенциала (более светлые области) носители фактически отсутствуют, в остальных 


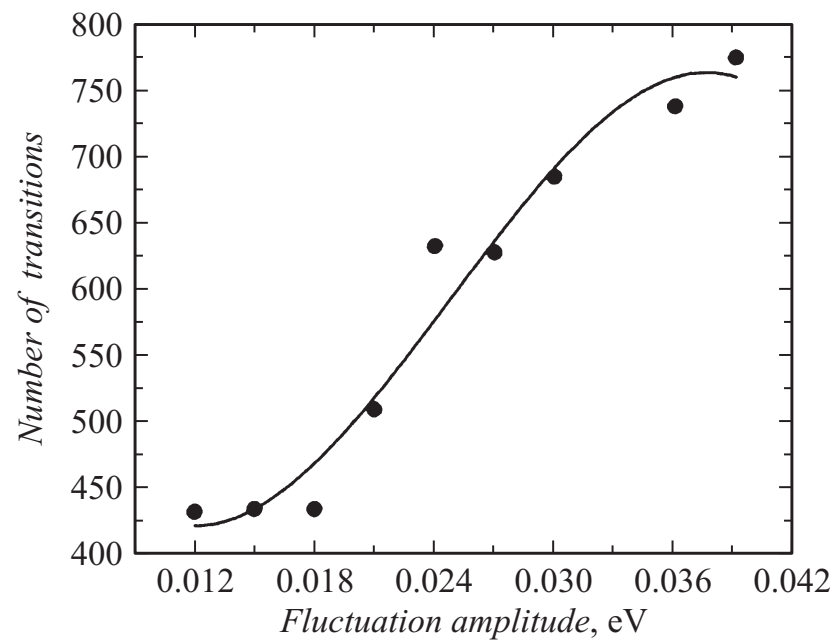

Pис. 5. Зависимость количества актов излучательной рекомбинации, произошедших в рассматриваемой модели за единицу времени, от амплитуды флуктуаций.

же областях, имеющих среднее значение потенциала, носители заметно более разрежены, нежели в ямах. Для случая рис. $4, b$ видно, что носители значительно более равномерно распределены по площади образца. Таким образом, при увеличении амплитуды флуктуаций носители распределяются по площади образца все более неравномерно, а значит, растет их локальная концентрация в области потенциальных ям. Это проявляется в увеличении количества актов излучательной рекомбинации, происходящих в единицу времени (рис. 5).

На рис. 6 приведены спектры люминесценции, сгенерированные при помощи построенной модели для различных амплитуд флуктуаций (после генерации спектров они были подвергнуты сглаживанию путем усреднения по 5 соседним точкам). Каждый показанный на рисунке спектр содержит информацию по примерно десяти тысячам излучательных переходов. На рис. $6, a$, где амплитуда флуктуаций для различных спектров отличается не столь значительно, видно, что при увеличении амплитуды флуктуаций пик спектра сдвигается в сторону меньших энергий, поскольку увеличение глубины потенциала ведет к уменьшению локального значения ширины запрещенной зоны и соответственно энергии испущенного кванта. Также видно, что с увеличением амплитуды флуктуаций линия излучения значительно уширяется, что объясняется пропорциональным увеличением разброса тех локальных минимумов энергии, из которых происходит основная люминесценция. Интегральная интенсивность излучательной рекомбинации при этом остается неизменной.

На рис. 6, $b$ приведены рассчитанные спектры люминесценции для случая, когда масштаб флуктуаций более значителен, чем на рис. $6, a$. Отметим, что все большее увеличение масштаба флуктуаций ведет к тому, что форма спектров, рассчитанных для образцов тех небольших, микронных размеров, которые приемлемы для счета на персональной ЭВМ, становится непредсказуемой (по существу такой расчет является аналогом микрофотолюминесценции). На длинноволновой части модельного спектра могут проявляться дополнительные пики, их появление обусловлено „высвечиванием“ отдельных областей образца, в которых флуктуационные потенциальные ямы имеют наибольшую глубину. Локализация носителей в таких потенциальных ямах (примерно $80 \%$ носителей находятся на $20 \%$ площади образца) приводит к „высвечиванию“ таких областей, что для нитридных материалов было экспериментально продемонстрировано, например, в [26]. Однако в экспериментах по ФЛ твердых растворов КРТ этот эффект пока обнаружен не был - особенности длинноволновой части спектров, наблюдавшиеся в ряде наших экспериментов, до сегодняшнего дня удавалось хорошо объяснить переходами носителей из зоны проводимости на акцепторные состояния в запрещенной зоне [9]. Коротковолновая часть спектра и в модели, и в низкотемпературном эксперименте по форме остается неизменной и является частью гауссовской кривой. Наиболее
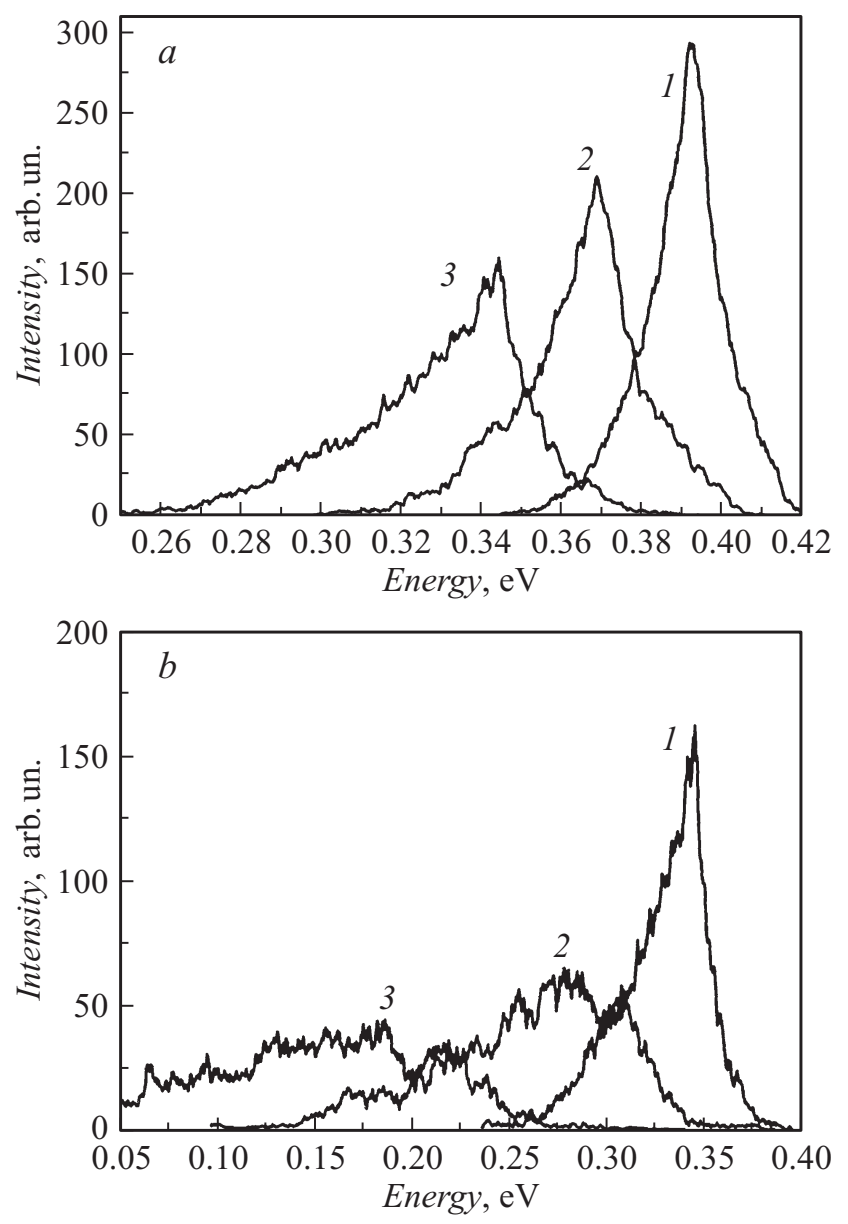

Рис. 6. Сравнение спектров ФЛ, рассчитанных в рамках модели для образца $\mathrm{CdHgTe}$ с $x=0.38$ при $T=5 \mathrm{~K}$, при различных амплитудах флуктуаций (в $\mathrm{eV}): a-1-0.016$, $2-0.022,3-0.028, b-1-0.035,2-0.070,3-0.115$. 
близко к экспериментально наблюдавшимся (см. рис. 2) оказалась величина полуширины линии излучения для расчетного спектра 1 на рис. $6, a-18.5 \mathrm{meV}$, она соответствует усредненному масштабу флуктуаций в $16 \mathrm{meV}$. На данном этапе, однако, попытки количественного описания масштаба флуктуаций в рамках модели были бы преждевременными в силу необходимости учета слишком большого числа факторов в эксперименте, как обсуждается выше. Тем не менее можно заключить, что на качественном уровне построенная модель вполне адекватно описывает влияние флуктуаций состава на излучательную рекомбинацию в рассматриваемых материалах, в частности на форму спектров люминесценции и энергетическое положение их пика.

\section{Заключение}

В работе проанализированы спектры фотолюминесценции гетероэпитаксиальных структур узкозонных твердых растворов $\mathrm{CdHgTe}$ и установлено наличие в изученных структурах масштабных флуктуаций состава, локализующих носители заряда. В программной среде MatLAB реализована физическая модель, использующая принципы волновых пакетов и рассматривающая процессы релаксации и рекомбинации фотовозбужденных носителей в среде с масштабными флуктуациями потенциала. Модель адекватно описывает влияние флуктуаций состава на излучательную рекомбинацию в подобных материалах: ее скорость, форму спектров люминесценции и положение их пика и дает хорошее согласие с экспериментальными данными. Результаты работы могут быть использованы при разработке фотоприемников и светоизлучающих структур среднего инфракрасного диапазона.

А.А. Грешнов благодарит за поддержку грант РФФИ 14-02-01223.

\section{Список литературы}

[1] Mattheis J., Rau U., Werner J.H. // J. Appl. Phys. 2007. Vol. 101. N 11. P. 113519.

[2] Grundmann M., Dietrich C.P. // J. Appl. Phys. 2009. Vol. 106. N 12. P. 123521.

[3] Li Q., Xu S.J., Xie M.H., Tong S.Y. // J. Phys. Cond. Matter. 2005. Vol. 17. N 30. P. 4853-4858.

[4] Schubert E.F., Gobel E.O., Horikoshi Y., Ploog K., Quiesser H.J. // Phys. Rev. B. 1984. Vol. 30. N 2. P. 813820.

[5] Клочихин А., Резницкий А., Тенишев Л., Пермогоров С., Иванов С., Сорокин С., Муманис Х., Сейсян Р., Клингширн С. // Письма ЖЭТФ. 2000. Т. 71. Вып. 6. С. 348-353.

[6] Lei W., Antoszewski J., Faraone L. // Appl. Phys. Rev. 2015. Vol. 2. N 4. P. 041303.

[7] Сидоров Ю.Г., Варавин В.С., Дворецкий С.А., Михайлов Н.Н., Якушев М.В., Сабинина И.В. // ФТП. 2001. Т. 35. Вып. 9. С. 1092-1101.
[8] Ivanov-Omskii V.I., Bazhenov N.L., Mynbaev K.D. // Phys. Stat. Sol. B. 2009. Vol. 246. N 8. P. 185-186.

[9] Мынбаев К.Д., Баженов Н.Л., Иванов-Омский В.И., Михайлов Н.Н., Якушев М.В., Сорочкин А.В., Ремесник В.Г., Дворецкий С.А., Варавин В.С., Сидоров Ю.Г. // ФТП. 2011. Т. 45. Вып. 7. С. $900-907$.

[10] Мынбаев К.Д., Бажсенов Н.Л., Шиляев А.В., Дворещкий С.А., Михайлов Н.Н., Якушев М.В., Ремесник В.Г., Варавин В.С. // ЖТФ. 2013. Т. 83. Вып. 10. С. 147-150.

[11] Ижснин И.И., Ижниин А.И., Мынбаев К.Д., Баженов Н.Л., Фицыч Е.И., Якушев М.В., Михайлов Н.Н., Варавин В.С., Дворецкий С.А. // ФТП. 2014. Т. 48. Вып. 2. С. 207-211.

[12] Мынбаев К.Д., Шиляев А.В., Баженов Н.Л., Ижнин И.И., Ижснин А.И., Михайлов Н.Н., Варавин В.С., Дворецкий C.A. // ФТП. 2015. Т. 49. Вып. 3. С. 379-384.

[13] Becker C.R., Latussek V., Pfeuffer-Jeschke A., Landwehr G., Molenkamp L.W. // Phys. Rev. B. 2000. Vol. 62. N 15. Art. 10353.

[14] Tomm J.W., Herrmann K.H., Yunovich A.E. // Phys. Stat. Sol. A. 1990. Vol. 122. N 1. P. 11-42.

[15] Fuchs F., Koidl P. // Semicond. Sci. Technol. 1991. Vol. 6. N 12C. P. C71-C75.

[16] Lusson A., Fuchs F., Marfaing Y. // J. Cryst. Growth. 1990. Vol. 101. N 1-4. P. 673-677.

[17] Gille P., Herrmann K.H., Puhlmann N., Schenk M., Tomm J.W., Werner L. // J. Cryst. Growth. 1988. Vol. 86. N 1-4. P. 593-598.

[18] Барановский С.Д., ЭФрос А.Л. // ФТП. 1978. Т. 12. Вып. 11. C. 2233-2237.

[19] Oudjaout D., Marfaing Y. // Phys. Rev. B. 1990. Vol. 41. N 17. P. 12096-12105.

[20] Stringfellow G.B. // J. Cryst. Growth. 2010. Vol. 312. N 6. P. 735-749.

[21] Шевченко Е.А., Жмерик В.Н., Мизеров А.М., Ситникова А.А., Иванов С.В., Торопов А.А. // ФТП. 2012. Т. 46. Вып. 8. С. 1022-1026.

[22] Liao Y., Kao C., Thomidis C., Moldawer A., Woodward J., Bhattarai D., Moustakas T.D. // Phys. Stat. Sol. C. 2012. Vol. 9. N 3-4. P. 798-801.

[23] Абакумов В.Н., Перель В.И., Яссиевич И.Н. // Безызлучательная рекомбинация в полупроводниках. СПб.: Изд-во ПИЯФ РАН, 1997. 376 с.

[24] Krishnamurthy S., Berding M., Yu Z.G. // J. Electron. Mater. 2006. Vol. 35. N 6. P. 1369-1378.

[25] Mynbaev K.D., Shilyaev A.V., Bazhenov N.L., Izhnin A.I., Izhnin I.I., Voitsekhovskii A.V., Mikhailov N.N., Varavin V.S., Dvoretsky S.A. // Физ. мех. матер. 2014. Т. 21. Вып. 1. C. $112-118$.

[26] De S., Layek A., Raja A., Kadir A., Gokhale M.R., Bhattacharya A., Dhar S., Chowdhury A. // Adv. Function. Mater. 2011. Vol. 21. P. 38283835. 